\title{
Resource Allocation in Cloud computing: A Comparative study
}

\author{
${ }^{1}$ Bhawna Dhruv, Payal , ${ }^{2}$ Praveen Kumar \\ M.Tech $(C S \& E)^{1,2}$, Assistant Professor ${ }^{3}$ Amity University Noida ${ }^{1,2,3}$
}

\begin{abstract}
Cloud computing is summed up as a different model for allowing favorable, network as per demand to use shared devices of computational resources which are collected and then released with marginal management effort or interaction with any client or any service provider. Cloud main objective is to cogitate on the demand of provision of the IT infrastructure. Cloud Computing tends to play fundamental role in different fields using new ideas, virtual world, e-business, networking engines used for searching on the web. It is used in ongoing settings where they are created without giving importance to a common programming model or take away of applications.
\end{abstract}

\section{Introduction}

For a start, cloud computing cannot be described as a technology but a prototype of provision and marketing IT sector that meet certain demands [1].Cloud is all about computer services and not only products. A few points that sum up cloud as a whole:

1) Distributed: The model proposed by cloud is distributed. Many clients use similar technology platform and also a particular application and its use.

2) Flexibility: The application of clouds are flexible. According to users, services are scalable so that the growth is limitless.

3) Pay as per need: Instead of paying according to plan which is given to every user in the traditional model, here the payment is done according to usage by user which is calculated as per time that can be different for every user.

4) Three Groups: The Cloud Computing is divided into three groups: Connectivity, Applications and Storage. Every segment has a predefined protocol and provides different protocol for business and organizations around the world.

The study that analyses the extent of usage for cloud computing services in various business organization is very critical. The study stresses upon vertical sections like different types of financial services, utilities and public sector. The study is said to observe current trends taking place to cloud computing and the extent of usage adoption for this different technology through various vertical sectors. It has also been helping to derive forecast of upcoming trends of adoption in cloud computing [2]. Cloud computing is a newly growing concept from different programming, hosting and management of web based survey links and data reporting in various applications. Cloud Computing is in great demand now-a-days due to minimum cost involved in the project as a whole. The cost here may refer in terms of infrastructure cost, computing cost etc. It provides virtual business to organizations so that different resources can be managed efficiently [3]. The most critical feature provided by cloud is the virtualization. Virtualization helps in differentiating hardware from that of software. The client can simply work on their applications with the help of multiple resources without worrying about the other running jobs. Due to this, the business organizations need not worry about updating the software or hardware as a cloud provides most updated versions of all dedicated resources [4]. Cloud computing makes it easier for the clients to use the services as per needs and also pay for them accordingly, thus providing great advantages like reduced cost, flexibility and scalability. Cloud can be typically divided into three types:

1) Public Cloud: As the name suggests, this type of cloud is available to general public or users. Different type of organization tends to choose this cloud so as to reduce the cost. However, there are issues in this type of cloud like security and management of data.

2) Private Cloud: As the name suggest, this type of cloud is dedicated to a single organization. And the services offered by this cloud can only be exploited by that particular business organization. This type of cloud is much more secure in terms of data.

3) Hybrid Cloud: This cloud is combination of both public and private cloud. This type of mixture expands deployment needs and hence increases the demand for such a cloud so as to fulfill the temporary needs of an organization which could be achieved in less time.

The motivation of this paper is to understand the scope of cloud computing and further study the frameworks of resource allocation. So as to find a solution for a problem like finite resources due to which the resources demanded by the clients cannot be met easily. So this becomes a major issue to satisfy the user in terms of requirement and working environments so that the profits are maximum. 


\section{Resource Allocation}

Resource Allocation can be defined as efficiently distributing the resources among multiple users as per their demands for given period of time. However, resource allocation has proven to be bit complicated in cloud computing. Therefore, there is a need to increase the computing capability for allocating the resources. The main aim of smartly allocating the resources is to gain financial profits in the market. This technique also boosts up the objectives of cloud computing i.e. pay as per use because the client need not pay for the resources that he has not used [5]. Dynamic resource allocation shoots up the work flow implementation and allows the users to differentiate among different policies available. Resource allocation strategy must avoid the following issues:

1) Over provisioning: This means that an application receives more amount of resources than actually demanded.

2) Under provisioning: This means that an application receives less amount of resources than actually demanded.

3) Contention of resources: This means that different applications try to access a single resource at same time.

4) Scarcity: This means there lack of resources.

Shown below is Table 1 that explains the required input for both the service provider and the client.

\begin{tabular}{|l|c|c|}
\hline Parameter & Provider & Customer \\
\hline Provider Offerings & $\sqrt{ }$ & - \\
\hline Resource Status & $\sqrt{ }$ & - \\
\hline Available Resources & $\sqrt{ }$ & - \\
\hline Application Requirements & - & $\sqrt{ }$ \\
\hline Agreed Contract & $\sqrt{ }$ & $\sqrt{ }$ \\
\hline
\end{tabular}

Table 1: Input Parameters

Due to limited resources various restrictions and increasing demands from the users, there is a need to efficiently allocate the resources to fulfill cloud requirement. The demand and supply of resources ma be available, hence there arises a need for different strategies that allocate the resource smartly. Given below are few strategies that analyses the issue of resource allocation in cloud computing.

1) Rule Based Resource Allocation:

To reduce the maintenance cost of resources, the resources allocation algorithm negotiates between multiple users to provide safer access to the resource across a network. Any failure in efficient negotiation may lead to the failure of whole cloud system. In RBRA, the distribution of resources is dynamic and the utilization of resources is at its peak [6]. The resources are allocated based upon the priority and hence a queue is formed, If any resource ' $\mathrm{R}$ ' is being used by and any user $\mathrm{X}$, and another user $\mathrm{X}$ demands it, Similarly user $\mathrm{Z}$ demands it any another time instant, then this algorithm creates a queue, giving the priority to $Y$ and then $Z$. This further means after $X$ has used the resource, $Y$ will use it and then $Z$ will use it. This increases the performance of the whole system. The priority may also be divided on the basis of task size. If the criticality of task is least, it is assigned last place. After the resource are allocated, the execution of tasks place and results are given to client.

2) Optimized Resource Scheduling:

This algorithm is based on the infrastructure as a services .i.e. Iaas. To provide best results, cloud computing makes use of virtual machine and in this algorithm, the virtual machine is distributed amongst many users so as to maximize the resource usage. An improved genetic algorithm is used here so as to allocate the resources in finest possible way [7]. The figure 1 shown below explains the resource scheduling in cloud computing.

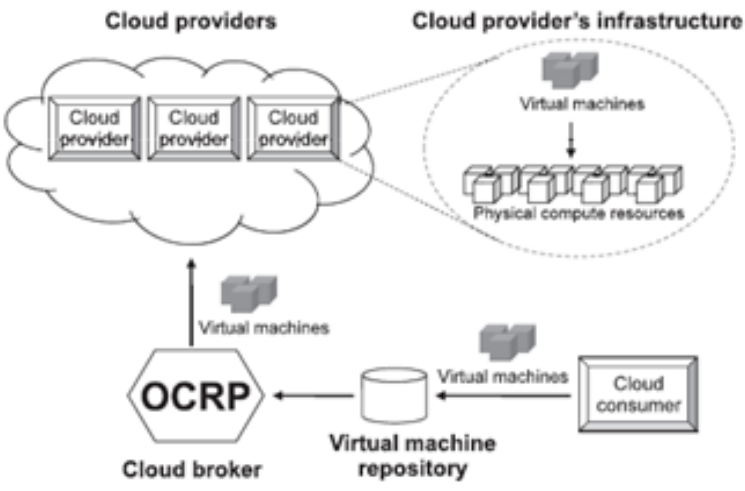

Fig 1: Resource Scheduling 
3) Fair Resource Allocation for Congestion Control:

Whenever resources are being allocated to any user or any service, definitely there are chances of congestion over the network. Congestion is a big problem as it deplete the overall performance, hence must be controlled. The FRA allows fair use of resources among different users because the need of resources may vary from every user. In this technique, whenever any user demands a particular service, a particular bandwidth is selected and is allocated to the client for a particular period of time [8]. After zero resources are left, all the new requests from a customer are rejected. Figure 2 explains the fair allocation of resources.

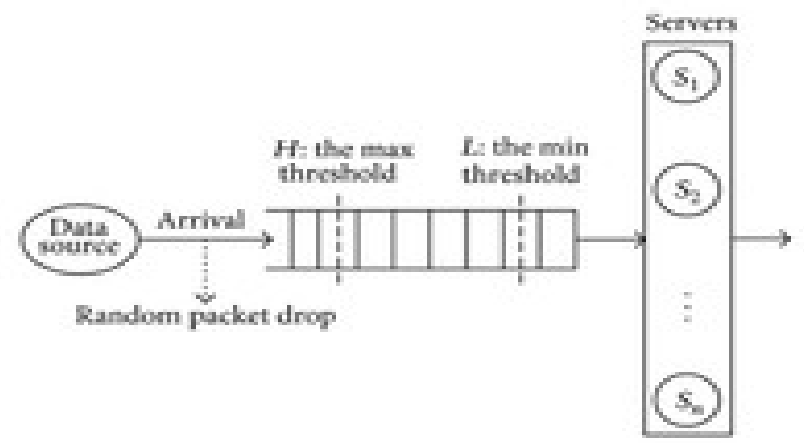

Fig 2: Fair Resource Allocation

4) Federated Computing and Network System:

In this model, both computing resources and network resources are mixed together. Therefore, for a combination of resources, FCNS is required. The synchronization of resources whether compute or network are altogether presented to FCNS which makes use of wavelength division multiplexing and offers best data transfer with least traffic over the network [9].

\section{Pros And Cons}

Given below are both pros and cons of the cloud computing as well as resource allocation technique used in this. Listed below are the pros of the model:

1) Cloud has proved to be more advantageous in terms of updating the hardware or the software. Any client need not update worry about the resources they are using [10].

2) There is overall access of the resources and application around the world.

3) There is an easier application of group as all the applications are available over internet and the use can exploit the same.

4) The computing power definitely increases if the resources are well allocated across the network.

5) Unlimited data storage is provide by the cloud, which also makes exploitation of resources and data easier for the users.

Listed below are the cons of the model:

1) As the data stays with the third party always, therefore there is no guarantee that the critical data is always safe. Due to this there is no worldwide acceptance of clod computing yet.

2) Sometimes due to compatibility issues, your input or output devices may not work well with cloud.

3) If any user wants to swap their cloud provider, then it might turn to be a difficult and time consuming process [11].

4) As all the data resides on the net, there is a possibility of loss of data due to phishing attacks or other such attacks.

5) Deep and exact knowledge is required while allocating the resources this may seem to be a difficult task.

The Table 2 shown below concludes the paper in a tabular form by comparing the critical issues of each resource allocation technique. The table further summarizes the above explained techniques:

\begin{tabular}{|c|c|c|c|c|}
\hline Parameters & RBRA & ORS & FRA & FCNS \\
\hline Bandwidth & High & Medium & High & Medium \\
\hline Cost & Less & Less & Less & Medium \\
\hline $\begin{array}{c}\text { Resource } \\
\text { Utilization }\end{array}$ & Optimal & High & Optimal & High \\
\hline $\begin{array}{c}\text { Technique } \\
\text { Followed }\end{array}$ & $\begin{array}{c}\text { Queue } \\
\text { System }\end{array}$ & GA & $\begin{array}{c}\text { Concurre } \\
\text { nt }\end{array}$ & WDM \\
\hline
\end{tabular}

Table 2: Comparision Of Techniques 


\section{Conclusion}

Cloud Computing has emerged as a powerful model for business models. It is an expanded network of computers that provide virtual business to any organizations. In this paper, we studied about what cloud computing is and the significance of resource allocation in cloud. If the allocation is done smartly, then definitely there is an increase in the computation power and hence provide fruitful results [12]. Cloud computing is in great demand due to numerous advantages that it has been offering. The different technologies discussed in the paper encourage us to study more algorithms to provide best allocation models to the clients as a future work.

\section{References}

[1] VVinothina,Dr.RSridaran,DrPadmavathiGanpathi,"A Survey on Resource Allocation in Cloud Computing", IJACSA, Vol3, No.6,2012.

[2] M Asad Afreen, Krzysztof Pawlikowski, Andears Willig,” A Framework for Resource Allocation Strategies in Cloud Computing Environment", 35th IEEE Annual Computer Software and Applications Conference Workshop, 2011.

[3] Jianpeng Dai, Bin Hu, Lipeng Zhu, Haiyun Han, Jianbo Liu," Research on Dynamic Resource Allocation with Cooperation Strategy in Cloud Computing, 3rd International Conference on System Science, Engineering and Manufacturing Informatization, 2012.

[4] N R Ram Mohan, E Baburaj, "Resource Allocation Techniques in Cloud Computing- Research Challenges for Applications", 4th International Conference on Computational Intelligence and Communication Network, 2012.

[5] Aman Kumar, Emmanuel S Pilli, R C Joshi,” An Efficient Framework for Resource Allocation in Cloud Computing”, IEEE, 31661.

[6] Swapnil M Parikh," A Survey on Cloud Computing Resource Allocation Techniques", Nirma University International Conference on Engineering, 2013.

[7] Pratik P Pandya, Hitesh A Bheda, "Dynamic Resource Allocation Techniques in Cloud Computing", International Journal of Advance Research in Computer Science and Management Studies, Vol 2, Issue 1, January 2014.

[8] K C Gouda, Radhika T V, Akshatha M,'Priority Based Resource Allocation Model for Cloud Computing", InternationalJournal of Science, Engineering and Technology Research, Vol2, Issue1, January 2013.

[9] Ronak Patel, Sanjay Patel, "Survey on Resource Allocation Strategies in Cloud Computing”, International Journal of Engineering and Research Technology, Vol 2, Issue 2, February 2013.

[10] K Prasanna Kumar, S Arun Kumar, Dr Jagadeeshan, "Effective Load Balancing for Dynamic Resource Allocation in Cloud Computing", International Journal of Innovative Research in Computer and Communication Engineering,Vol 2 , Issue 3,March 2013.

[11] Yang Wt Al,"A Profile Based Approach to Just in Time Scalability for Cloud Applications', IEEE International Conference, 2009.

[12] Peter Mell, Timothy Grance," The NIST Definition of Cloud Computing (Draft)", Computer Security Division, Information Technology Laboratory, 2011. 